\section{Where are we in the battle of ending tuberculosis in children and adolescents in South Afirica?}

To the Editor: Ambitious targets to end tuberculosis (TB) were set at the United Nations General Assembly High-Level Meeting (UNHLM) on TB in September 2018, with children and adolescents specifically noted as key populations deserving of more attention. ${ }^{[1]}$ In addition, the World Health Organization (WHO) launched a revised 'Roadmap towards ending TB in children and adolescents, ${ }^{[2]}$ outlining key actions that should be taken at country level to engage relevant stakeholders to optimally prevent and treat TB in these age groups.

In South Africa (SA), paediatric TB notifications $(<15$ years) declined steeply between 2015 and 2017 (29 137 in 2015, 20546 in 2016 and 15628 in 2017). ${ }^{[3]}$ This fall could be due either to a true decline in cases or to a smaller proportion of cases being found. In 2018, SA reported 17561 cases to the $\mathrm{WHO},{ }^{[3]}$ a $12 \%$ increase from 2017 and probably due to the national 'finding the missing TB cases campaign' that was implemented in SA in 2018.

The WHO uses mathematical modelling to estimate the TB burden at both global and national level. In 2018, the WHO estimated that 27000 children ( $<15$ years; $95 \%$ confidence interval 18000 - 36 000) developed TB in SA. ${ }^{[3]}$ Despite the 2018 increase in notifications, these estimates suggest that the SA childhood TB case detection rate is still only $65 \%$, leaving a third of children with TB in SA undiagnosed or unreported.

At the UNHLM, SA committed to diagnose and treat 95500 children between 2018 and 2022 (Fig. 1). ${ }^{[4]}$ Although SA achieved $96 \%$ of its target for 2018 , services will need to be strengthened if it is to keep up with these commitments and improve case detection. To achieve this, the country will need better diagnostics for young children, non-invasive and point-of-care microbiological sampling and testing for all children, and more training to empower healthcare workers to make clinical diagnoses. Following diagnosis, it is also essential to ensure accurate and complete reporting of all cases. Sub-national analyses of routine TB data can additionally provide information on where interventions are most needed.

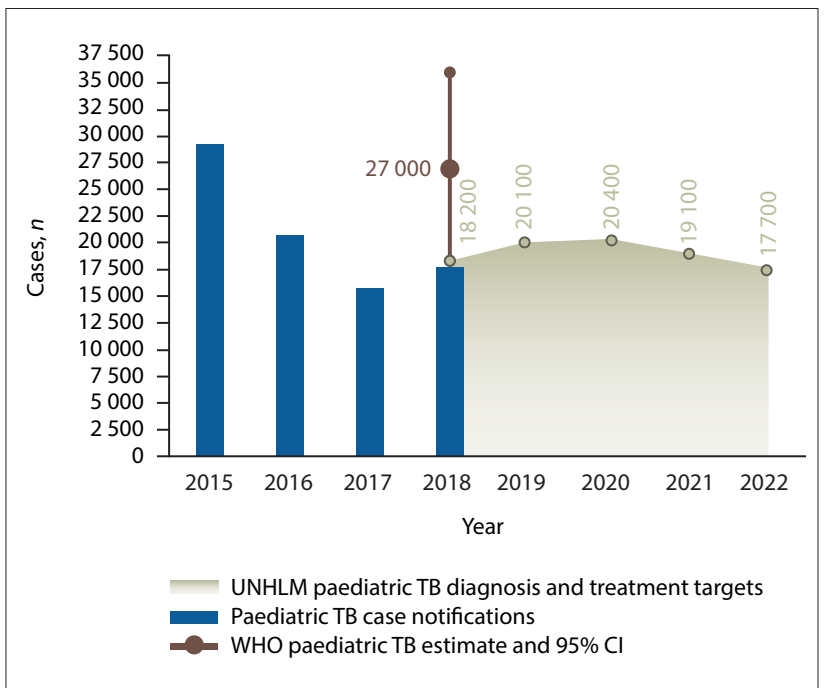

Fig. 1. National paediatric ( $<15$ years) TB case notifications, WHO burden of disease estimates and committed UNHLM paediatric TB diagnosis and treatment targets for South Africa. $(\mathrm{TB}=$ tuberculosis; $W H O=$ World Health Organization; UNHLM = United Nations General Assembly HighLevel Meeting; $C I=$ confidence interval.)
TB preventive therapy (TPT) is a safe and effective strategy to prevent TB disease in children following exposure. ${ }^{[5]}$ At the UNHLM, SA committed to provide TPT to 206510 child contacts aged $<5$ years. ${ }^{[4]}$ SA is shortly to roll out shorter TPT regimens, and if this approach is coupled with strengthened TB contact management and PT implementation, ${ }^{[6]}$ it could drastically reduce the burden of $\mathrm{TB}$ disease among children and adolescents.

The lack of surveillance data on adolescents (10 - 19 years) with TB remains a concern. Despite being recognised as a vulnerable group, they are 'missing' in the age bands currently reported, being included either with children in the 5-14-year age band or with adults in the 15 - 24-year age band. ${ }^{[7]}$ SA has a strong TB surveillance system, allowing age-disaggregated reporting at a much more granular level. The country should either revise the current age bands or report adolescents separately if service provision to this age group is to be evaluated properly.

On 24 March, we commemorated World TB Day. Each year, this represents an opportunity to reflect on the promises made for children and adolescents, to evaluate progress, and to identify future priorities.

Author contributions. KdP and JAS developed the idea and produced the first draft. All authors reviewed the drafts and provided valuable input. All authors reviewed and approved the final version.

Funding. KdP is supported by the Fogarty International Center of the National Institutes of Health under Award No. K43TW011006. The content is solely the responsibility of the authors and does not necessarily represent the official views of the National Institutes of Health. ACH is financially supported by the South African National Research Foundation (NRF) South African Research Chairs Initiative (SARChI). KdP is partly supported by this NRF SARChI grant to ACH. The financial assistance of the NRF towards this research is hereby acknowledged. The opinions expressed and conclusions arrived at are those of the authors and are not necessarily to be attributed to the NRF.

Conflicts of interest. None.

\section{K du Preez}

Desmond Tutu TB Centre, Department of Paediatrics and Child Health, Faculty of Medicine and Health Sciences, Stellenbosch University, Cape Town, South Africa karen_dupreez@sun.ac.za

\section{J A Seddon}

Desmond Tutu TB Centre, Department of Paediatrics and Child Health, Faculty of Medicine and Health Sciences, Stellenbosch University, Cape Town, South Africa; and Department of Infectious Diseases, Imperial College London, UK

\section{H S Schaaf}

Desmond Tutu TB Centre, Department of Paediatrics and Child Health, Faculty of Medicine and Health Sciences, Stellenbosch University, Cape Town, South Africa; and Department of Paediatrics and Child Health, Tygerberg Hospital, Cape Town, South Africa

\section{A C Hesseling}

Desmond Tutu TB Centre, Department of Paediatrics and Child Health, Faculty of Medicine and Health Sciences, Stellenbosch University, Cape Town, South Africa 
1. Political Declaration of the United Nations General Assembly High-Level Meeting on Ending TB 26 September 2018, New York. Geneva: World Health Organization, 2018. https://www.who.int/tb/ unhlmonTBDeclaration.pdf (accessed 16 March 2020).

2. World Health Organization. Roadmap towards ending TB in children and adolescents. 2nd ed Geneva: WHO, 2018. http://apps.who.int/iris/bitstream/handle/10665/275422/9789241514798-eng.

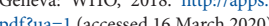

3. World Health Organization. TB Data: WHO’s global tuberculosis database 2019. https://www.who.int/ tb/data/en/ (accessed 30 March 2020).

4. United Nations High-Level Meeting on TB: Country targets for childhood TB diagnosis and treatment. http://www.stoptb.org/resources/countrytargets/ (accessed 16 March 2020).

h. http:/ Ww.stopt.org/resources/country: Corgets/ accessed $16 \mathrm{M}$. Ayieko J, Abuogi L, Simchowitz B, Bukusi EA, Smith AH, Reingold A. Efficacy of isoniazid prophylactic therapy in prevention of tuberculosis in children: A meta-analysis. BMC Infect Dis 2014;14:91. https:/
6. Szkwarko D, Hirsch-Moverman Y, du Plessis L, du Preez K, Carr C, Mandalakas AM. Child contact management in high tuberculosis burden countries: A mixed-methods systematic review. PLoS One 017:12(8):e0182185. https://doi.org/10.1371/journal.pone. 0182185

7. Snow KJ, Cruz AT, Seddon JA, et al. Adolescent tuberculosis. Lancet Child Adolesc Health 2020;4(1):68-79. https://doi.org/10.1016/S2352-4642(19)30337-2

S Afr Med J 2020;110(5):345-346. https://doi.org/10.7196/SAMJ.2020.v110i5.14728 